\title{
„Grundrente“: Koalitionskitt mit Folgen
}

Vor fast 20 Jahren wurde der Umbau des deutschen Alterssicherungssystems als Jahrhundertreform gefeiert. Durch einen Niveauabbau in der gesetzlichen Rentenversicherung (GRV) sollte eine kapitalmarktabhängige private und betriebliche Sicherung gefördert werden. Diese Reform wurde unter anderem deshalb kritisiert, weil eine steigende Altersarmut befürchtet wurde. Die damaligen Verfechter der neuen Politik bestritten dies allerdings vehement. Doch schon in der übernächsten Legislaturperiode legten Regierung und Opposition Vorschläge für eine Aufwertung von Renten langjährig Versicherter vor - als "Lebensleistungs-“ oder „Solidarrente“ bezeichnet und 2013 wurden diese dann als "solidarische Lebensleistungsrente" in den Koalitionsvertrag übernommen. Doch erst jetzt hat sich die neuerliche große Koalition zu einem Kompromiss durchgerungen - wohl als Kitt für den Zusammenhalt der Koalition.

Bereits die 2005 eingeführte steuerfinanzierte, bedarfsabhängige „Grundsicherung im Alter und bei Erwerbsminderung" sollte nach dem Willen der damaligen rot-grünen Bundesregierung faktisch mit der GRV verschmolzen werden. Dies hätte die GRV aus Sicht der Versicherten immer mehr von einem beitragsfinanzierten, am früheren Lebenshaltungsniveau anknüpfenden System zu einem Umverteilungsinstrument verändert, zumal die GRV auch die Einkommensprüfung durchführen sollte. Doch Widerstände führten schließlich dazu, dass die vorgesehene Bedürftigkeitsprüfung - ähnlich wie in der Sozialhilfe - heute nicht in der GRV, sondern nach wie vor in einem separaten System geregelt wird. Nun gibt es einen neuen Anlauf, eine Anhebung niedriger Renten langjährig Versicherter ohne Bedürftigkeitsprüfung durchzusetzen, eine Forderung der SPD. Diese neue „Grundrente“ soll für langjährig Versicherte höher sein als die bedürftigkeitsgeprüfte Grundsicherung und zur Vermeidung von Altersarmut beitragen. Und sie soll - wie schon früher gefordert - in die GRV integriert werden.

Zwar setzte die SPD nicht alle ihre Vorstellungen vollständig durch, aber weitgehend. Es sei ihr gelungen, die CDU/CSU „nach allen Regeln der Kunst über den Tisch zu ziehen", war in der Frankfurter Allgemeinen Zeitung zu lesen. Zwar soll es keine Bedürftigkeitsprüfung geben, aber - wie die CDU-Vorsitzende die Unterschiede kaschierend formulierte - eine „Bedürftigkeitsprüfung in Form einer umfassenden Prüfung des Einkommens". Das Einkommen soll nun wiederum innerhalb der GRV geprüft werden. Dies wirft mannigfache Fragen auf - im Zusammenhang mit der Abgrenzung des Personenkreises (für den Altersarmut vermieden werden soll), der Ermittlung des Grundrentenbetrags und der dafür erforderlichen Einkommensüberprüfung, der Finanzierung und auch für das Konzept der GRV.

Die jetzt vorgelegte und nicht einfach nachzuvollziehende Ausgestaltung der neuen „Grundrente" sieht ab 2021 vor, dass bei mindestens 35 Jahren mit bestimmten Versicherungszeiten (zu denen unter anderem auch Kindererziehungs- und Pflegezeiten zählen, jedoch keine Zeiten der Arbeitslosigkeit) in der GRV angesammelte individuelle „Entgeltpunkte“ (EP), sofern sie zwischen 0,3 und 0,8 liegen (was Ende $2019 \mathrm{im}$ Westen einer Rente zwischen rund 347 Euro und 925 Euro entspricht), für maximal 35 Jahre aufgewertet werden. Hierzu soll die Zahl der individuellen EP maximal verdoppelt werden, jedoch nicht auf mehr als 0,8, d.h., die Erhöhung ist zwischen 0,3 und 0,4 EP umso stärker, darüber jedoch umso niedriger, je höher die individuellen EP sind. Der dabei errechnete Betrag soll dann um 12,5\% gekürzt werden - „zur Stärkung des Äquivalenzprinzips“. Aber ohne Begründung wirkt dies wie ein Feigenblatt, falls dies nicht allein finanzpolitisch motiviert ist.

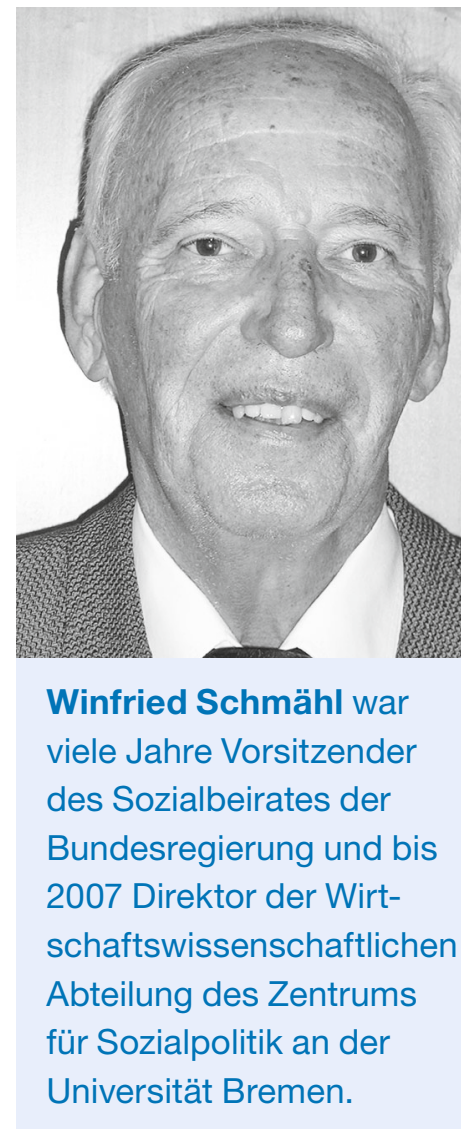


Für den zu begünstigenden Versichertenkreis wird dann geprüft, ob das Einkommen - hierzu zählt das zu versteuernde Einkommen zuzüglich „des steuerfrei gestellten Anteils der Rente und aller Kapitalerträge“ - bestimmte Freibeträge übersteigt. Falls ja, so werden darüberliegende Beträge auf die Grundrente angerechnet. Die Einkommensprüfung soll durch einen Datenabgleich zwischen Rentenversicherung und Finanzämtern erfolgen, ist aber angesichts organisatorischer Fragen noch weitgehend ungeklärt.

Zwar sollen die zusätzlichen Ausgaben im Prinzip - was aber wachsweich formuliert wurde - aus Steuern finanziert werden. Doch inwieweit das tatsächlich geschieht, steht noch in den Sternen. Erfahrungen aus jüngerer Zeit (so z. B. mit der Finanzierung der sogenannten „Mütterrente“, die dann statt aus Steuern aus Beiträgen finanziert wird) lassen befürchten, dass das Konzept der beitragsfinanzierten, am früheren Erwerbseinkommen orientierten Renten immer weiter unterhöhlt wird. Allerdings wird dies - wie schon bei der Reform des Jahres 2001 - von manchen Interessenvertretern und Wissenschaftlern geradezu begrüßt. Da schließlich alles im Rahmen der GRV umgesetzt werden soll, ist nicht nur zu befürchten, dass aus praktischen Gründen immer weiter von einer umfassenden Einkommensprüfung abgerückt wird, sondern vor allem, dass die GRV aus Sicht der Beitragszahler zunehmend als (interpersonelles) Umverteilungssystem wahrgenommen wird, bei dem die zu erwartende Rente immer weniger von den zuvor gezahlten Beiträgen abhängt. Darunter dürfte die Abgabenbereitschaft der Arbeitnehmer leiden und die Beitragspflicht eher als Anreiz zum Ausweichen in die Schattenwirtschaft wirken. Man bedenke, dass individuelle Entgeltpunkte unabhängig von der Arbeitszeit aufgewertet werden, die dem Lohn zugrunde lag. Die Verteilungseffekte des nun vorgesehenen Maßnahmenpakets sind alles andere als einfach zu durchschauen. Vorgesehen ist auch ein Freibetrag für GRV-Renten derjenigen, die bereits die Grundsicherung im Alter beziehen und 35 Beitragsjahre aufweisen, wie auch ein Freibetrag beim Wohngeld. Dies alles ist nicht nur komplex, sondern manches bleibt noch weitgehend unklar. Hoffentlich haben das die Koalitionäre bei ihrer Entscheidung hinreichend durchschauen können.

Insgesamt gewinnt man den Eindruck, dass - nicht zuletzt im Interesse des Erhalts der gegenwärtigen Koalition - Kompromisse gesucht wurden, die jedoch viele Fragen aufwerfen. So sollen besonders niedrige Beiträge langjährig Versicherter aufgewertet, ihre „Lebensleistung“ anerkannt und damit auch Altersarmut bekämpft werden. Doch ob damit überhaupt in signifikanter Weise einen Beitrag zur Vermeidung von Altersarmut geleistet wird, ist angesichts der gewählten Rahmendaten (35 „Grundrentenjahre“) zu bezweifeln. Eher werden langfristige Folgen für das System der deutschen Alterssicherung deutlich, so ein Verwischen der Grenzen zwischen Beitragsfinanzierung - mit der ein Anspruch auf eine Gegenleistung erworben wird - und der Steuerfinanzierung, die ja nicht mit einem Anspruchserwerb verknüpft ist. Die GRV würde damit im Zweifel immer stärker von einem am früheren Lohn anknüpfenden Versicherungssystem zu einer primär auf Vermeidung von Altersarmut ausgerichteten „Basissicherung“. Eine sich am Lebenshaltungsniveau während des Erwerbslebens orientierende Alterssicherung würde dann primär Aufgabe privater und betrieblicher Einrichtungen sein, abgewickelt über den Kapitalmarkt. Welchen Interessen das dient, ist unschwer zu erkennen.

Als vor 20 Jahren die damalige Regierung eine bedarfsorientierte Mindestsicherung im Rahmen der GRV durchsetzen wollte, erfolgten angesichts massiver Kritik stattdessen Regelungen in der Grundsicherung bzw. der Sozialhilfe. Dieser Weg könnte auch jetzt wieder beschritten werden, damit es nicht - wie sonst zu befürchten ist

Winfried Schmähl

Universität Bremen w.schmaehl@t-online.de
- zu einer weiteren Verwässerung des Konzepts der einkommensbezogenen und beitragsfinanzierten GRV kommt, sondern eine klare Aufgabenteilung zwischen den Institutionen realisiert wird. 